\title{
ASIC/System Hardware Verification at Nortel: A View from the Trenches
}

\author{
A. Silburt \\ Hardware System Modeling Group \\ Nortel Semiconductors \\ 185 Corkstown Road \\ Nepean, Ontario K2H 8V/ Canada
}

\begin{abstract}
The emergence of functional verification as the largest single component of the ASIC development cycle has come as a bit of a surprise to the design community. At Nortel, we have had a group focusing on the methodologies used at this stage of the design for four years. Our involvement with some of the largest Nortel hardware designs has provided us with first-hand experience of the challenges in establishing confidence that ASICs will work the first time out in a system. This talk will describe the scope of the problems faced in ASIC-intensive telecomm systems, the methodologies currently deployed, and the emerging technologies that we are looking towards in the future to improve our ability to handle complex hardware systems.
\end{abstract}

